\title{
Evaluation of the Appropriateness of Gastrointestinal Prophylaxis in the Critically III
}

\author{
Kayla Torppey, Leena Kansagra, Sheetal Patel, Rouel Guiang \\ Department of Pharmacy, Newark Beth Israel Medical Center, Newark, NJ 070112 United States
}

\begin{abstract}
The aim of this study was to determine the utilization of SRMD (stress related mucosal disease) prophylaxis in the ICU (intensive care unit) of a large teaching institution based on duration and defined adverse effects. Patients were reviewed for appropriateness of SRMD prophylactic therapy based on the presence of two independent risk factors (coagulopathy and mechanical ventilation greater than 48 hours) versus presence of any one risk factor from a list developed by the study investigator. Data was collected into spreadsheets and outcomes were analyzed using descriptive statistics. When evaluating patients based on the presence of any risk factor, 84 percent of patients had at least one risk factor present, while 16 percent did not have any. In patients who received famotidine, there was one occurrence of Clostridium difficile and 6 cases of electrolyte abnormalities. In patients who received a PPI, there was one documented case of HAP, two cases of ventilator-associated pneumonia, three cases of Clostridium difficile, and 14 patients who developed electrolyte abnormalities. Data does not show an association between acid suppression therapy and incidence of nosocomial infections. The number of patients whose therapy exceeded the appropriate stop-date compared with the number of patients in which SRMD prophylaxis was discontinued when risk factors diminished was greater when evaluating patients based upon the two independent risk factors.
\end{abstract}

Key words: Stress ulcer prophylaxis, critical care, GI prophylaxis, proton pump inhibitor, H2RA.

\section{Introduction}

In critically ill patients, GI (gastrointestinal) bleeding resulting from stress ulceration is a complication that can occur. As a result, the use of acid suppression therapy for stress ulcer prophylaxis has become a routine practice in the ICU (intensive care unit). Issues with acid suppression therapy include the initiation of therapy without risk factors or continuation of therapy even after risk factors have diminished, leading to increased risks of infection, such as Clostridium difficile and hospital-acquired pneumonia [1].

SRMD (stress-related mucosal disease) in critically ill patients is thought to be caused by visceral hypoperfusion. Although the exact etiology is not completely understood, it is thought to be multifactorial, resulting from various physiologic

Corresponding author: Kayla Torppey, Pharm. D., BCPS, Newark Beth Israel Medical Center Email: ktorppey@barnabashealth.org. responses to the stress, including sympathetic system activation, increased catecholamine release, hypovolemia, decreased cardiac output, visceral vasoconstriction, increased abdominal compartment pressures, and the release of proinflammatory cytokines [2]. Moreover, stress related responses damage the integrity of the gastric mucosa by reducing gastrointestinal blood flow, oxygen delivery and bicarbonate secretion, compromising the mucosal barrier resulting in further damage to the epithelial layer. Additionally, gastrointestinal motility is decreased following splanchnic hypoperfusion, which delays the removal of acidic material from the stomach and prolongs exposure of the poorly defended mucosa to gastric acid, increasing the risk of ulceration [3].

Options for stress ulcer prophylaxis include sucralfate, H2RAs (histamine-2 receptor antagonists), and PPIs (proton pump inhibitors). H2RAs include such agents as cimetidine, ranitidine, famotidine, and nizatidine. H2RAs exert their action by decreasing 
gastric acid secretion through a reversible, competitive inhibition of histamine-stimulated acid secretion. The clinical adverse effects attributed to H2RAs in critically ill patients include thrombocytopenia (especially in children), confusion (especially in the elderly and renally impaired), interstitial nephritis, rapid infusion-related hypotension, sinus bradycardia, and possibly pneumonia. A large database analysis by Laheij and colleagues reported that users of H2RAs had a 1.63 -fold increased risk of pneumonia (95\% confidence interval, 1.07-2.48) compared with those who had discontinued use [4]. All H2RAs are eliminated renally to some extent and thus require dosage adjustments for renal dysfunction. Additionally, cimetidine is an inhibitor of cytochrome $\mathrm{P} 450$ isoenzymes $3 \mathrm{~A} 4,2 \mathrm{D} 6,2 \mathrm{C} 9,2 \mathrm{C} 19$, and $1 \mathrm{~A} 2$ which can make utilization challenging $[3,5]$. On the other hand, PPIs such as pantoprazole, lansoprazole, and omeprazole work by inhibiting the final step in acid production via the parietal cell $\mathrm{H}+/ \mathrm{K}+$ ATP pump. Due to this mechanism of action, they provide long-lasting suppression of acid secretion and are the most potent antisecretory agents available. Furthermore, PPIs suppress acids for greater than 20 hours, permitting once-daily dosing without requiring gastric $\mathrm{pH}$ monitoring. All PPIs are hepatically eliminated by cytochrome $\mathrm{P} 450$ isoenzymes $3 \mathrm{~A} 4$ and $2 \mathrm{C} 19$. Increased risk of fractures, pneumonia, and Clostridium difficile infection have been attributed to PPI therapy, but a definitive relationship has yet to be established [6-8]. Finally, sucralfate works by coating the gastric mucosa, creating a thin, protective layer between the mucosa and the gastric acid in the lumen. In an acidic environment, it forms a polymer that eventually binds with the protein cations in the exposed ulcer. Since it is not systematically absorbed, it may decrease the absorption of other concomitantly administrated oral medications such as ciprofloxacin, phenytoin, and digoxin to name a few. Some adverse effects associated with sucralfate are constipation and aluminum toxicity [3-6].
Critically ill patients are at increased risk of developing a stress ulcer and consequent bleeding as a result of underlying disease and medical interventions [3]. In a prospective, multicenter cohort study of 2252 patients admitted to ICUs performed by Cook and colleagues, two strong, independent risk factors for stress-related mucosal bleeding were identified. These risk factors included respiratory failure requiring mechanical ventilator support for more than 48 hours and the presence of a coagulopathy. Coagulopathy was defined as a platelet count $<50,000 \mathrm{~mm}^{3}$, an International Normalization Ratio of $>1.5$ or a partial thromboplastin time $>2$ times the control value. ${ }^{1}$ Moreover, a meta-analysis conducted by Alhazzani and colleagues concluded that PPIs were more effective than H2RAs at reducing clinically important upper gastrointestinal bleeding $(\mathrm{p}=0.002)$ and overt upper gastrointestinal bleeding $(p<0.0001)$. However, of the 12 trials reviewed, only three were judged to be at low risk of bias and demonstrated a lower risk of GI bleeding with PPIs than with H2RAs [9].

Ultimately, the primary objective of this study is to determine the utilization of GI prophylaxis in patients on the medical service in the ICU to evaluate overuse, reduce adverse effects, and improve patient outcomes and care.

\section{Materials and Methods}

NBIMC (Newark Beth Israel Medical Center) is a 673-bed teaching institution that is home to the Barnabas Health Heart Center, which offers cardiology services and cardiac surgery programs. The hospital has an active heart transplant program, therefore, many patients at NBIMC are awaiting transplant and on ventricular assist devices.

A retrospective chart analysis of 150 patients admitted to the Intensive Care Unit at NBIMC was conducted from May 1, 2013 to July 31, 2014. Patients were included if they were 18 years of age or older, and had received pantoprazole, lansoprazole, famotidine, or sucralfate for SRMD prophylaxis in the ICU. Please 
refer to Table 1 for complete dosing information. Patients were excluded if they had an active GI bleed or received an organ transplant within one year. A list of patients meeting the aforementioned inclusion criteria were randomly selected for review. Each patient's Electronic Medical Record was reviewed through Cerner solutions software. All data was kept confidential, and access was only granted to study personnel. This data was recorded using Microsoft Excel $^{\circledR}$ spreadsheets and evaluated. Patients were reviewed for appropriateness of SRMD prophylactic therapy based on the presence of either of the two independent risk factors versus the presence of any one risk factor using the following schematic (Fig. 1).

Renal failure was defined as a creatinine clearance less than $40 \mathrm{~mL}$ per minute, oliguria $(<500 \mathrm{~mL}$ of urine per day), or a serum creatinine greater than 2.8 mg per deciliter. If a patient had any two of the following: a serum bilirubin concentration greater than $8.8 \mathrm{mg}$ per deciliter, a serum aspartate aminotransferase level of greater than $500 \mathrm{U}$ per liter, or a serum albumin level less than $41 \mathrm{~g}$ per liter, and clinical signs and symptoms of hepatic coma they were deemed to have hepatic failure. Coagulopathy was defined as a platelet count less than $50,000 \mathrm{~mm}^{3}$, an INR (international normalization ratio) of more than 1.5 , or a partial thromboplastin time greater than two times the control value. Severe sepsis was defined as sepsis-induced hypotension, lactate above upper limits laboratory normal, urine output less than $0.5 \mathrm{~mL} / \mathrm{kg} /$ hour

Table 1 Definitions for appropriate dosing for SRMD prophylactic therapy.

\begin{tabular}{|l|l|}
\hline Prophylactic Agent & Dose \\
\hline Famotidine & $\begin{array}{l}\mathrm{CrCl} \geq 50 \mathrm{~mL} / \text { minute: } 20 \mathrm{mg} \text { twice daily } \\
\mathrm{CrCl}<50 \mathrm{~mL} / \text { minute: } 50 \% \text { dose reduction }\end{array}$ \\
\hline Lansoprazole & $30 \mathrm{mg}$ once daily \\
\hline Pantoprazole & $40 \mathrm{mg}$ once daily \\
\hline Sucralfate & $1 \mathrm{~g}$ four times daily (before meals and at bedtime) \\
\hline
\end{tabular}

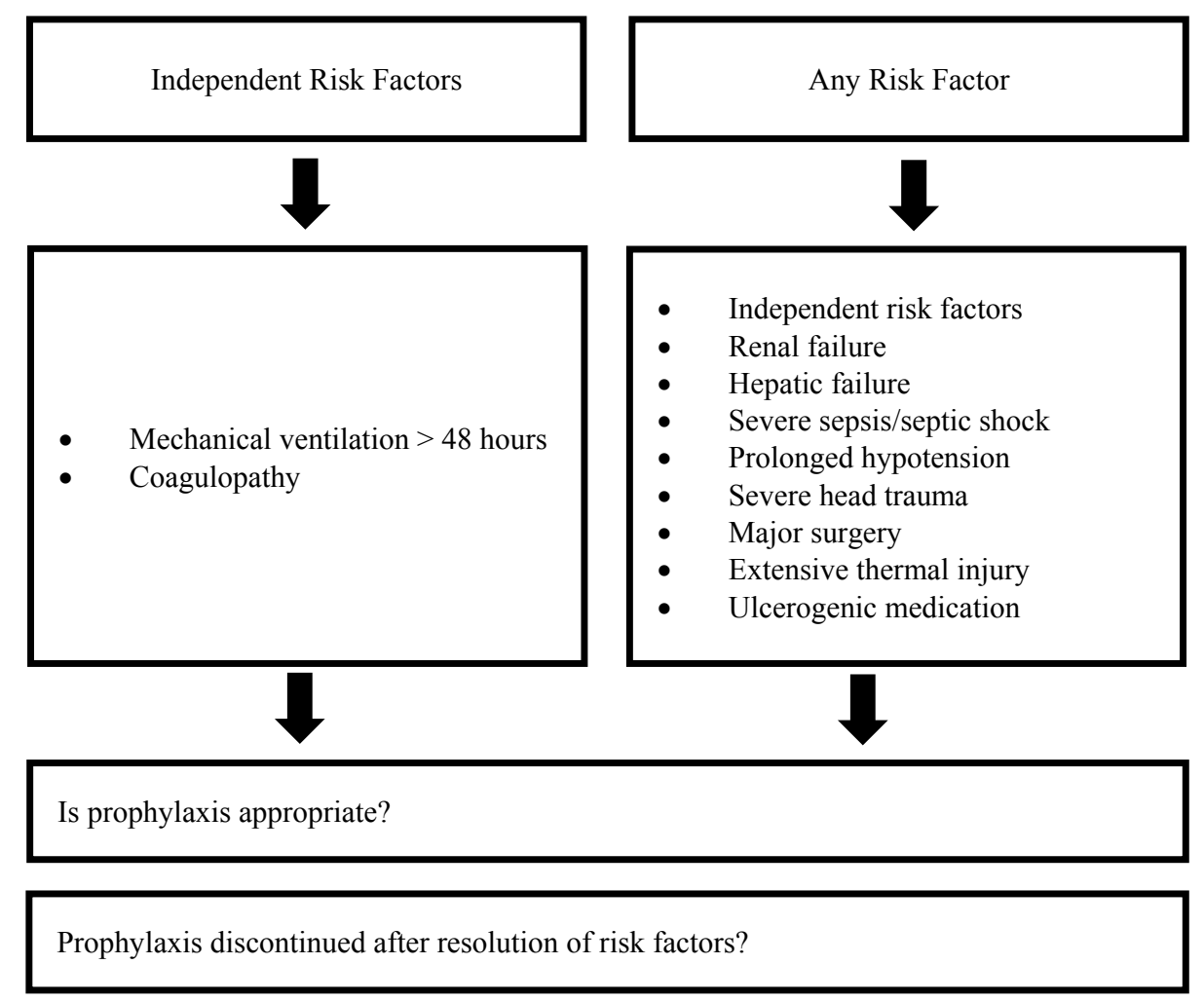

Fig. 1 Evaluation of therapy. 
for more than 2 hours despite adequate fluid resuscitation, acute lung injury with Pao2/Fio2 less than 250 in the absence of pneumonia as infection source, acute lung injury with Pao2/Fio2 less than 200 in the presence of pneumonia as infection source, creatinine greater than $2.0 \mathrm{mg} / \mathrm{dL}$, bilirubin above 2 $\mathrm{mg} / \mathrm{dL}$, platelet count less than 100,000, and an INR greater than 1.5 , whereas the criteria for septic shock met that of severe sepsis, but included hypotension, despite adequate fluid resuscitation. Study investigators defined hypotension as systolic blood pressure less than $80 \mathrm{mmHg}$ and severe head trauma as a Glasgow coma score less than 8. Furthermore, major surgery was defined as lasting more than 4 hours and extensive thermal injury needed to involve more than $35 \%$ of the body surface area to meet criteria. Finally, ulcerogenic medication was considered $250 \mathrm{mg}$ or more of hydrocortisone (or the equivalent) per day.

Analyses of the primary endpoints were performed using descriptive statistics. The following parameters were included in the data collection: patient demographics, medical diagnoses, laboratory values (such as renal function, hepatic function, and platelet function tests), the agent(s) used for prophylaxis, and duration of prophylactic therapy.

\section{Results}

150 patients were randomly selected, and 116 were evaluated for appropriateness of therapy. 34 patients were excluded due to GI bleed, transplant within one year, bowel resection surgery, and one patient never received therapy although prophylaxis was ordered. Of the 116 patients receiving prophylaxis, 53\% received therapy with an H2RA, 63\% with a PPI (either lansoprazole or pantoprazole), and $14 \%$ with sucralfate. However, 32 patients received more than one agent during their length of stay. This data is depicted in Fig. 2.

This study assessed the indication for SRMD prophylaxis based on risk factors for SRMD. As depicted in Fig. 3, when evaluating patients based on the presence of either of the two independent risk factors,

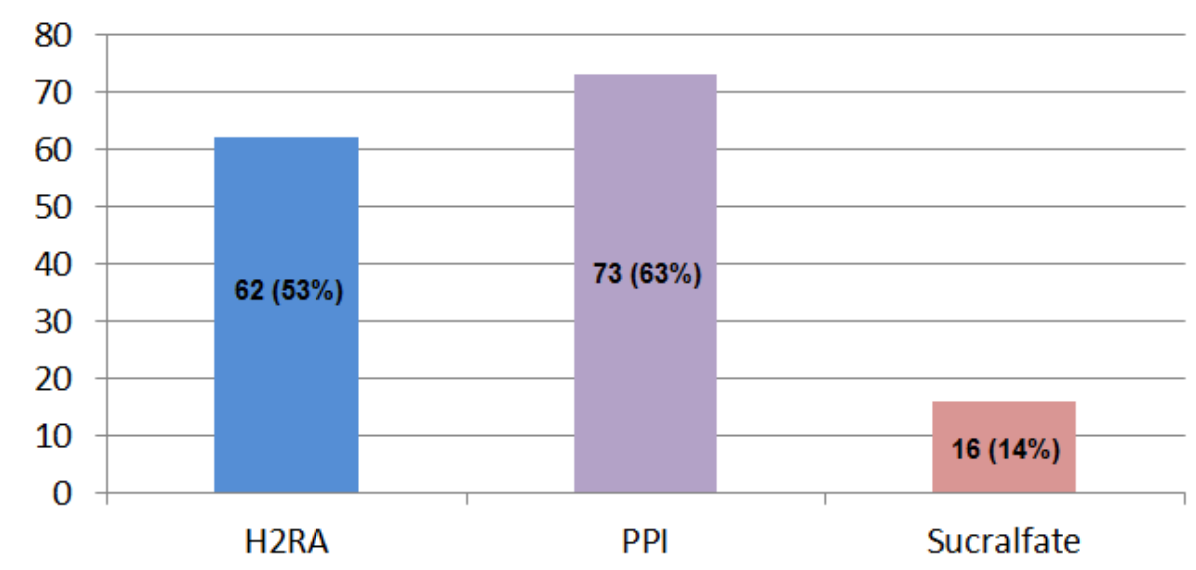

Fig. 2 Number of Patients Receiving Prophylaxis $(n=116)$.

Either Independent RF

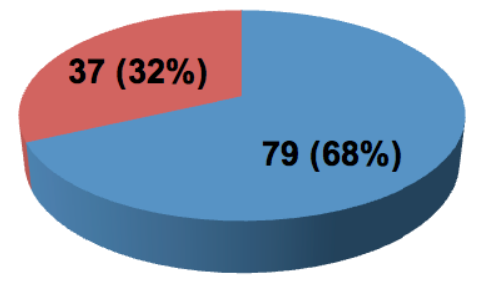

RF Present

RF Not Present
Any RF

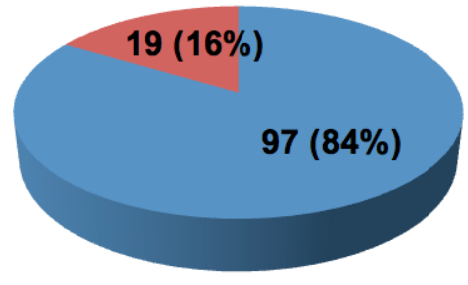

Fig. 3 Indications for Prophylaxis based on RF (risk factors). 
Table 2 Incidence of opportunistic infections, electrolyte abnormalities, and bleeding as a result of prophylaxis.

\begin{tabular}{lllll}
\hline & $\begin{array}{l}\text { H2RA } \\
(\mathrm{n}=62)\end{array}$ & $\begin{array}{l}\text { PPI } \\
(\mathrm{n}=73)\end{array}$ & $\begin{array}{l}\text { Sucralfate } \\
(\mathrm{n}=16)\end{array}$ & Days of Therapy, Median \pm IQR \\
\hline HAP & 0 & $1(1.4 \%)$ & 0 & $26 \pm 0$ \\
VAP & 0 & $2(2.7 \%)$ & 0 & $21 \pm 13$ \\
Clostridium difficile & $1(1.6 \%)$ & $3(4.1 \%)$ & 0 & $9.5 \pm 4.5$ \\
Electrolyte abnormality & $6(9.7 \%)$ & $14(19.2 \%)$ & 0 & $15 \pm 13.5$ \\
Bleeding & 0 & 0 & 0 & 0 \\
\hline Duration of therapy for all patients, Median + IQR $8 \pm 10$ & &
\end{tabular}

Duration of therapy for all patients, Median \pm IQR: $8 \pm 10$

79 patients had either mechanical ventilation or the presence of coagulopathy, while 37 patients did not. However, when evaluating patients based on the presence of any one risk factor, 97 patients had at least one present, while 19 did not have any risk factors for SRMD.

When analyzing the therapy received, the number of patients who were continued on prophylactic therapy beyond indication differed depending upon whether they were evaluated based on the presence of either of the two independent risk factors or the presence of any risk factor. When evaluating patients based on the presence of either two independent risk factors versus the presence of any risk factor, the percentage of patients whose prophylactic therapy continued beyond indication was $63 \%$ and $47 \%$, respectively. Moreover, the median \pm IQR days that prophylactic therapy continued beyond indication respectively was $1.5 \pm 6$ and $0 \pm 2$.

Although this was not one of our endpoints, data was collected for patients to assess whether they were on acid suppression therapy at home. A total of 38 patients (33\%) were on acid suppression therapy at home, but only 13 of those patients (34\%) had an appropriate indication noted in their chart. This study evaluated if patients were placed on an appropriate dosing regimen. Please refer to Table 1 for complete dosing information. In total, 21 patients (18\%) were on an inappropriate regimen, where 19 patients did not receive a renal dose adjustment when necessary and 2 patients received higher than prophylactic dosing.

Finally, the study trended the incidence of HAP, VAP, Clostridium difficile, electrolyte abnormalities, and bleeding with the prophylactic agent received and median days of therapy. In patients who received an H2RA, one patient developed Clostridium difficile and 6 patients developed electrolyte abnormalities. In patients who received a PPI, one patient developed HAP, 2 patients developed VAP, 3 patients developed Clostridium difficile, and 14 patients developed electrolyte abnormalities. Patients who were treated with sucralfate did not develop abnormalities or infection. Additionally, those patients who developed nosocomial infections or abnormalities had a longer median duration of therapy compared with all patients in the study. Full results are depicted in Table 2.

\section{Discussion}

While SRMD can double the length of an ICU stay, inappropriate continuation of acid suppression therapy in patients can also contribute to increased morbidity and cause negative outcomes. Results of this analysis demonstrated that there was not an association between SRMD prophylaxis and the incidence of infections, while there was an increased trend in occurrence with patients receiving PPIs. When comparing the duration of therapy for all patients in the study, the median days was greater in those that had documented infections and electrolyte abnormalities. The observed incidence of Clostridium difficile could possibly be attributed to antibiotic use in critically ill patients rather than a direct result of SRMD prophylactic therapy, but because this study was not designed to find an association, this cannot be concluded.

One limitation faced during the implementation of this study that should be considered upon interpretation 
of the results is the overall patient population. Due to the nature of the critically ill population, many patients in this study expired before being on SRMD prophylaxis long enough to see any long-term adverse effects. Moreover, many critically ill patients meet criteria for receiving SRMD prophylaxis due to the risk factors typically associated with their condition. It would be beneficial to analyze SRMD prophylaxis utilization in patients on general medicine floors, where they may be inappropriately continued on therapy after being downgraded from the ICU.

Currently, the American Society of Health-System Pharmacists has tentatively scheduled the release of their "Gastrointestinal Stress Ulcer Prophylaxis" guidelines to "Spring 2016." It will be interesting to see what recommendations are put forth by the society, and whether PPIs or H2RAs receive a more favorable endorsement.

\section{Conclusions}

When evaluating utilization of prophylactic therapy, appropriateness differed based upon which risk factors were being assessed. If considering the two independent risk factors, the number of patients whose therapy exceeded the appropriate stop-date was greater than when evaluating patients based on the presence of any risk factor.

In addition, data did not show a clear association between acid suppression therapy and incidence of pneumonia, Clostridium difficile, electrolyte abnormalities, or bleeding. However, there was a trend which demonstrated a higher incidence of adverse effects from those who received therapy with a PPI versus H2RAs and sucralfate. Further studies need to be conducted to evaluate patients with a longer length of stay, extending to general medicine floors. These results have been presented to the critical care fellows and attending physicians at NBIMC to provide education on limiting SRMD prophylactic therapy to only those who have either of the independent risk factors. By educating providers, medication therapy can be managed correctly and adverse events can be prevented. Ultimately, results will also be presented to other medical services, and future studies are being planned to analyze data in other units.

\section{Acknowledgements}

This research received no specific grant from any funding agency in the public, commercial, or not-for-profit sectors.

\section{Conflicts of interest}

The Authors declare that they have no conflicts of interest to disclose.

\section{References}

[1] Cook, D. J. et al. 1994. "Risk Factors for Gastrointestinal Bleeding in Critically Ill Patients. Canadian Critical Care Trials Group.” N. Engl. J. Med. 330: 377-81.

[2] Mutlu, G. M. et al. 2001. "GI Complications in Patients Receiving Mechanical Ventilation." Chest 119: 1222-41.

[3] Quenot, J., Thiery, N., and Barbar, S. 2009. "When Should Stress Ulcer Prophylaxis Be Used in the ICU?" Current Opinion in Critical Care 15: 139-43.

[4] Laheij, R. J. et al. 2004. "Risk of Community-Acquired Pneumonia and Use of Gastric-acid Suppressive Drugs." JAMA 292: 1955-60.

[5] Spirt, M. J., and Stanley, S. 2006. "Update on Stress Ulcer Prophylaxis in Critically Ill Patients." Crit. Care Nurse 26: 18.

[6] ASHP. 1999. "Therapeutics Guidelines on Stress Ulcer Prophylaxis.” Am. J. Health Syst. Pharm. 56: 347-79.

[7] Krag, M. et al. 2014. "Stress Ulcer Prophylaxis versus Placebo or no Prophylaxis in Critically Ill Patients: ASystematic Review of Randomized Clinical Trials with Meta-analysis and Trial Sequential Analysis." Intensive Care Med. 40 (1): 11-22.

[8] Farrell, C. P. et al. 2010. "Overuse of Stress Ulcer Prophylaxis in the Critical Care Setting and Beyond." J. Crit. Care 25 (2): 214-20.

[9] Alhazzani, W. et al. 2013. "Proton Pump Inhibitors Versus Histamine 2 Receptor Antagonists for Stress Ulcer Prophylaxis in Critically Ill Patients: A Systemic Review and Meta-analysis." Crit Care Med 41: 693-705. 